\title{
Nanocarbons in selective oxidative dehydrogenation reaction
}

\author{
D.S. Su ${ }^{\text {a, }}$, N. Maksimova a ${ }^{\text {, J.J. Delgado }}{ }^{\text {a }}$, N. Keller ${ }^{\text {b }}$, G. Mestl ${ }^{\text {c*** }}$, M.J. Ledoux ${ }^{\text {b }}$, R. Schlögl ${ }^{\text {a }}$ \\ ${ }^{a}$ Department of Inorganic Chemistry, Fritz-Haber-Institut of the Max Planck Society, Faradayweg 4-6, \\ D-14195 Berlin, Germany \\ ${ }^{b}$ Laboratoire des Mate'riaux, Surfaces et Prece'de's pour la Catalyse, Universite' Louis Pasteur, UMR 7515 \\ CNRS, 25 rue Becquerel, 67087 Strasbourg Cedex 02, France \\ ${ }^{c}$ NanoScape AG, Frankfurter Ring 193a, 80807 München, Germany
}

\begin{abstract}
Nanocarbons (multiwalled carbon nanotubes (CNTs) and onion-like carbon) are tested for the oxidative dehydrogenation (ODH) of ethylbenzene to styrene. The catalytic performances are compared with these using carbon black and graphite as catalysts. The tested nanocarbons are catalytic active in the reaction. The highest yield is achieved by using onion-like carbon as catalyst. The combustion stability of graphite, carbon nanotubes and onion-like carbons gives stable catalytic performances on stream. The comparison of onion-like carbons as a model catalyst with other carbon materials gives the criteria for efficient carbon catalyst. A reaction model for oxidative dehydrogenation on carbon catalysts is proposed too. Our findings provide a new perspective for the application of nanostructured carbon materials.
\end{abstract}

\section{Introduction}

Nanocarbons such as carbon nanotubes (CNTs) have attracted a lot of scientific interests worldwide, and research mainly concerns the synthesis, characterization tools and technological applications [1]. Among several potential applications including new field emitters or hydrogen storage, catalysis seems to be highly promising, and the use of carbon nanofibers and nanotubes as support or confinement vessel for catalytically active phases led to peculiar conversion/selectivity patterns for several reactions, summarized in recent reviews $[2,3]$.

The application of nanocarbons in the oxidative dehydrogenation of ethylbenzene to styrene will be discussed in this paper. The production of styrene monomer from ethylbenzene is one of the ten largest production processes in the chemical industry with an annual styrene production of 23 million tons [4,5]. Nowadays, the production of styrene is carried out by the endothermic

\footnotetext{
* Corresponding author.

E-mail addresses: dangsheng@fhi-berlin.mpg.de (D.S. Su), Gerhard_mestl@nanoscape.de (G. Mestl).

** Co-corresponding author.
}

thermal dehydrogenation (DH). This process is performed at high temperatures $\left(600-700^{\circ} \mathrm{C}\right)$ over an hematite catalyst promoted by potassium $(10 \%)$ and other compounds $\left(\mathrm{Al}_{2} \mathrm{O}_{3}\right.$, $\mathrm{Cr}_{2} \mathrm{O}_{3}, \mathrm{~V}, \mathrm{Ce}, \mathrm{W}, \mathrm{Mo}$ ) to enhance both the catalyst selectivity and lifetime [6]. A strong incentive for the development of alternative technologies arises from this energy intense DH process and its thermodynamic limitations, the use of large amounts of overheated steam and irreversible catalyst deactivation $[7,8]$. Numerous articles have pointed out that the oxidative dehydrogenation $(\mathrm{ODH})$ is an elegant and very promising alternative to conventional dehydrogenation $[8,9]$. The strongly exothermic ODH of hydrocarbons in the presence of oxygen reduces the need for heat supplied to the reactor, and the reaction is also less thermodynamically limited than the DH route. A variety of catalysts, including phosphates, alumina and other metal oxides showed activity for the ODH of ethylbenzene to styrene, as reviewed in by Cavani and Trifira [8]. Evidence was gradually accumulated that the active sites were not located on the initial catalyst surface, but on a carbonaceous overlayer.

More recently, carbon itself, in particular activated carbon, showed activity for the ODH reaction [9-12]. In the series of papers by Figueiredo and co-workers [10-12], it is found that an increased amount of carbonyl/quinone groups 
on the surface of activated carbon leads to an improved performance of the catalyst. A redox mechanism involving quinone/hydroquinone groups on the surface of the activated carbon is suggested for the reaction [11]. However, the activated carbon catalysts displayed a low stability as a function of time on stream in an oxidative atmosphere, hindering the potential use of activated carbons as catalyst [12]. Carbon nanofilaments and onion-like carbon are reported also to be active in the $\mathrm{ODH}$ of ethylbenzene to styrene $[13,14]$.

This article deals with the use of carbon nanotubes as catalyst for the $\mathrm{ODH}$ of ethylbenzene to styrene and with the comparison of the catalytic performance of CNTs, onion-like carbon, carbon black and graphite. The hypothesis is put forward that the nature and microstructure of the carbon material determine a high and stable catalytic performance. This is the basis for a suggested structureactivity relationship. Comparison was made between carbon black, graphite, carbon nanotube and onion-like carbon catalysts.

\section{Experimental section}

\subsection{Carbon catalysts}

Carbon black (lamp black, $26 \mathrm{~m}^{2} / \mathrm{g}$ ) and high surface area graphite (HSAG $300,267 \mathrm{~m}^{2} / \mathrm{g}$ ) were supplied by Degussa (Düsseldorf, Germany) and Timcal (Bodio, Switzerland), respectively. The multi-walled carbon nanotubes (CNTs, $26 \mathrm{~m}^{2} / \mathrm{g}$ ) used for the present work were purchased from Applied Science Inc. (OH, USA) and produced by catalytic hydrocarbon decomposition. They had a mean outer diameter of $90 \mathrm{~nm}$. Onion-like carbons (OLC, $456 \mathrm{~m}^{2} / \mathrm{g}$ ) were produced by thermal annealing of ultra-dispersed diamond (UDD, $297 \mathrm{~m}^{2} / \mathrm{g}$ ) powder at $2143 \mathrm{~K}$ under a $10^{-6}$ torr vacuum as described by Kuznetsov et al. [15], and consisted of 5-10 nm diameter concentric graphene shell structures with a content of more than $95 \%$ of $\mathrm{sp}^{2}$-hybridized carbon.

\subsection{Characterization techniques}

In order to understand the catalytic properties of the sample, fresh and used catalysts were characterized by different bulk and surface sensitive techniques. Transmission electron microscopy (TEM) analysis was performed with a Phillips CM200 FEG field-emission gun electron microscope operating at an accelerating voltage of $200 \mathrm{kV}$. The samples were prepared by suspending the solid powder in ethanol under ultrasonic vibration. One drop of the prepared suspension was brought onto holey carbon films on copper grids.

X-ray photoelectron spectroscopy (XPS) was carried out on a modified Leybold Heraeus spectrometer (LHS12 MCD) with $\mathrm{Mg} \mathrm{K} \alpha$ radiation $(1253.6 \mathrm{eV}$ ) and a power of $240 \mathrm{~W}$.
The bandpass energy was set to $50 \mathrm{eV}$. X-ray satellites and Shirley backgrounds were subtracted. The peak areas were normalized with the theoretical cross-sections to obtain the relative surface compositions. The $\mathrm{C} 1 \mathrm{~s}$ and $\mathrm{O} 1 \mathrm{~s}$ peaks were fitted by combined convoluted Gauss Lorentz functions.

\subsection{Catalytic tests}

The performances of carbon catalysts were investigated at temperatures between 790 and $823 \mathrm{~K}$. The catalytic reaction was carried out in a quartz tube reactor $(4 \mathrm{~mm}$ i.d. $\times 200 \mathrm{~mm}$ ), holding $0.02 \mathrm{~g}$ of catalyst particles between two quartz wool plugs in the isothermal zone. Ethylbenzene was evaporated at $35^{\circ} \mathrm{C}$ (ethylbenzene partial pressure of $2160 \mathrm{~Pa}$ ) in flowing $\mathrm{He}$, and subsequently mixed with oxygen, corresponding to an ethylbenzene concentration of 2 vol. $\%$ and an ethylbenzene-oxygen ratio of $1: 1$. The total flow was $10 \mathrm{ml} / \mathrm{min}$, i.e. a liquid hourly space velocity (LHSV) of $0.5 \mathrm{~h}^{-1}$. The inlet and outlet gas analysis was performed using an on-line gas chromatograph equipped with two columns for simultaneous analysis of aromatics and permanent gases: a $5 \%$ SP-1200/1.75\% bentone 34 packed column for the hydrocarbons and a carboxen 1010 PLOT column for the permanent gases, coupled to FID and TCD detectors, respectively.
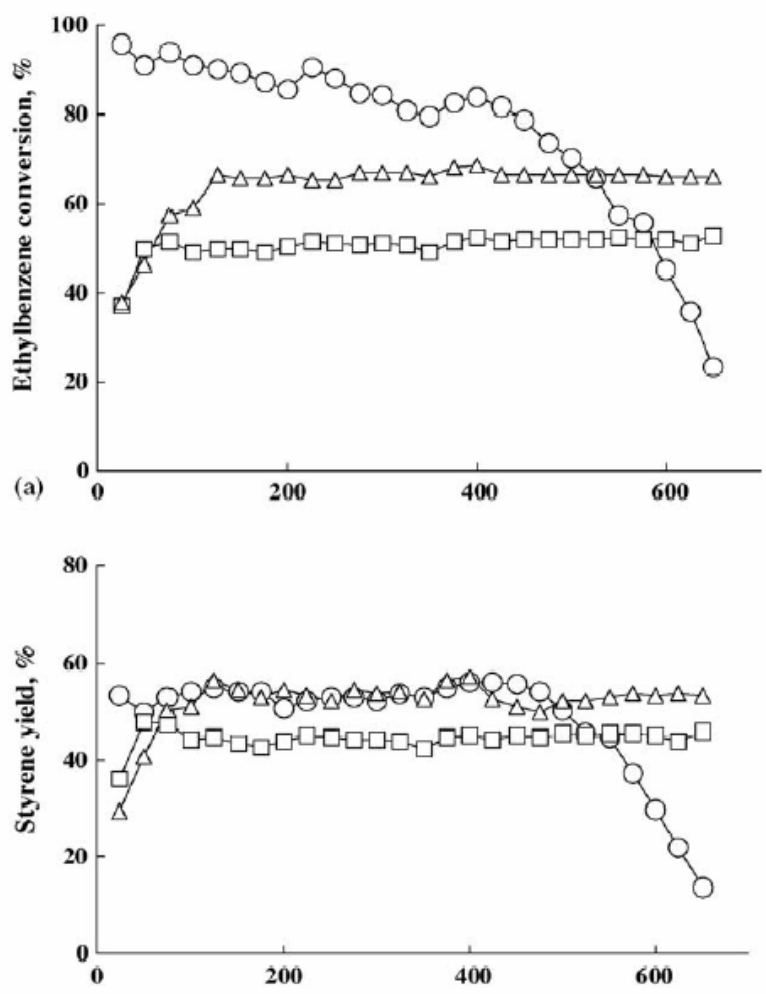

(b)

Time on stream, min

Fig. 1. Ethylbenzene conversion (a) and styrene yield (b) as a function of time on stream obtained at $823 \mathrm{~K}$ on carbon black $(O)$, graphite $(\square)$ and CNTs $(\triangle)$. 


\section{Results and discussion}

\subsection{Carbon black, graphite and carbon nanotubes}

Fig. 1 shows the catalytic performances of carbon black, graphite and CNTs in the ODH of ethylbenzene. Carbon black exhibited a high activity at the beginning of the reaction, with an initial ethylbenzene conversion of $96 \%$ and a selectivity to styrene of $53 \%$, resulting in a styrene yield of $51 \%$. However, its catalytic activity continuously decreased with time on stream due to the combustion of carbon black that was completely burned off after $720 \mathrm{~min}$ on stream. Hence, the catalytic behavior of carbon black paralleled its instability, which renders it useless as catalyst in $\mathrm{ODH}$ reaction. On the other hand, the graphite and CNT catalysts displayed a different behavior and showed stable performances with time on stream, after an initial activation period on stream of about 2 and $3 \mathrm{~h}$, respectively. The CNTs and graphite catalysts exhibited a steady-state ethylbenzene conversion of 64 and $52 \%$, respectively, leading to a styrene yield of 54 and $44 \%$, respectively. However, it has been proven that CNTs were stable toward combustion under severe oxidative reaction conditions in long time experiments, i.e. 3 days. This stability/unstability of carbon catalysts could be related to the stability/unstability toward the combustion [16] of the different carbon materials.

Fig. 2 displays two high-resolution TEM images of CNT walls before and after the ODH reaction. The wall of the fresh CNTs consisted of two layers (Fig. 2a). An inner layer of conical graphite layers, with an angle of the inclination of the graphite layers to the nanotube axis of about $26^{\circ}$ and interplane distance of $0.348 \mathrm{~nm}$ close to that of graphite, was covered by highly disordered carbon layers oriented parallel to the tube axis with an average interplane distance of about $0.388 \mathrm{~nm}$, forming the outer nanotube shell. After $20 \mathrm{~h}$ of time on stream, this external shell had disappeared and was replaced by $\mathrm{sp}^{2}$ carbon with short-range ordering, especially at the edges of the conical graphene sheets (Fig. 2b). It has been proposed that this carbon layer was deposited during the reaction in line with the unclosed carbon balance observed on CNTs. Additionally, the ends of the conical layers seem to be partially oxidized [16]. According to these observations, the induction period could be related to the combustion of the highly disordered carbon. These partially oxidized prism faces were presumably formed during the induction period and can play an important role in the catalytic reaction. Hence, the high catalytic activity was only developed after the graphite layers were accessible to the gas phase.

\subsection{Onion-like carbons}

In order to obtain more insight into the structure-activity relationship and the roll of oxygen groups in the DOH, OLC was considered as a valuable tool due to a clean and welldefined oxygen-free surface. OLC thus can be seen as a

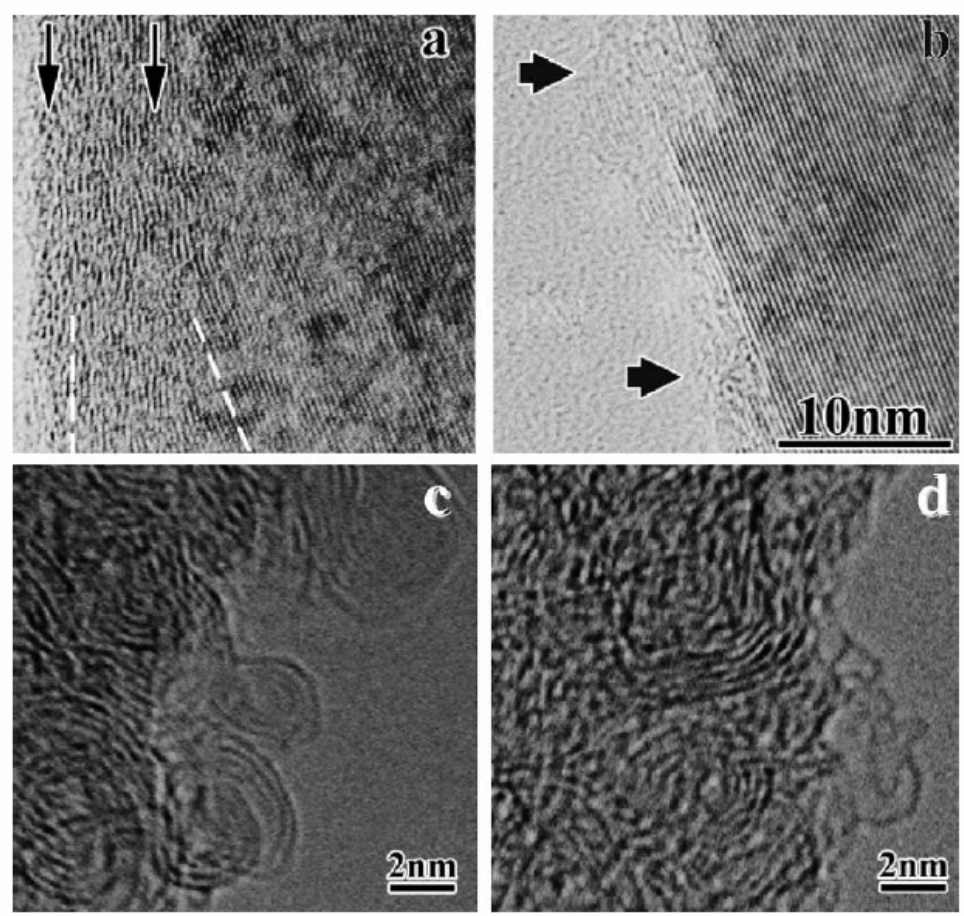

Fig. 2. High-resolution TEM images of the CNT wall before the reaction (a) and after $20 \mathrm{~h}$ on stream (b) and OLC micrographs before (a) and after (b) the ODH reaction. 


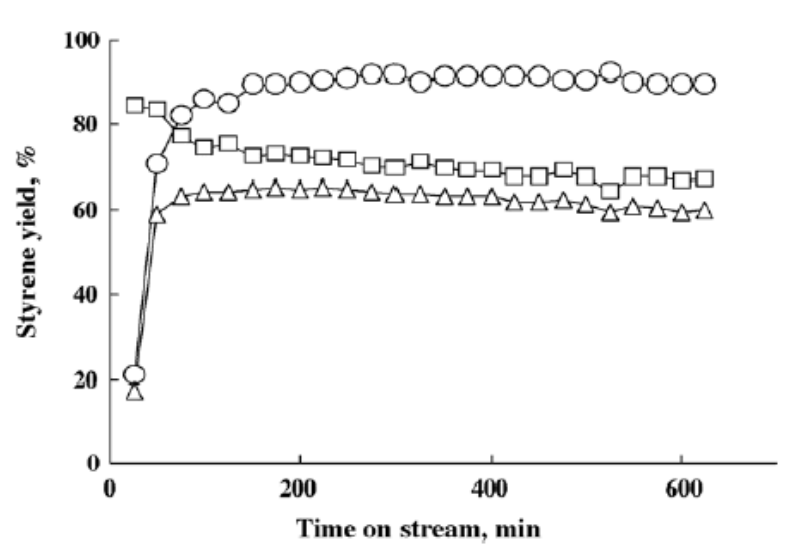

Fig. 3. Ethylbenzene conversion ( $\bigcirc)$, selectivity to styrene $(\square)$ and styrene yield $(\triangle)$ as a function of time on stream obtained on OLC at $790 \mathrm{~K}$.

representative "model system". Fig. 3 shows the catalytic behavior of the OLC material with time on stream at $790 \mathrm{~K}$. The OLC catalyst exhibited an activation period of about $2 \mathrm{~h}$, from a minor initial activity to a conversion level of $92 \%$ at the steady-state. An almost stable styrene selectivity allowed a high yield of $62 \%$. High-resolution TEM images of both fresh and used OLC are shown in Fig. 2. The difference in contrast for the used OLC (Fig. 2d) when compared to the image of the fresh multishell OLC (Fig. 2c) confirmed the formation of ill-defined carbon during the reaction. The radical increase of the catalytic activity should result from a significant restructuring leading to the generation of novel functional groups onto the OLC surface.

The C 1s XPS spectra after reaction (Fig. 4a) indicated the presence of the oxidized carbon by an increased intensity at the high-energy wing of the $\mathrm{C}$ 1s signal between 285 and $290 \mathrm{eV}$. The O 1s spectra (Fig. 4b) confirmed that the oxygen-free carbon surface of the fresh OLC was transformed after reaction into an oxygen-containing surface (dotted line) and provided information about their chemical nature. The deconvolution of the $\mathrm{O} 1 \mathrm{~s}$ spectrum after reaction revealed three different chemical environments for oxygen, which could be assigned to $\mathrm{C}=\mathrm{O}(530,7 \mathrm{eV}),-\mathrm{OH}$ $(532.5 \mathrm{eV})$ and adsorbed water $(533.8 \mathrm{eV})$ [17,18]. The dehydrogenating power of the catalyst thus seems to be linked to the generation of the strongly basic $\mathrm{C}=\mathrm{O}$ sites during activation period [14]. The presence of $\mathrm{OH}$ groups can indicate that the mechanism involves carbonyl-quinone/ hydroxyl and styrene/ethylbenzene redox couples. This was previously suggested by Figuereido et al. using different activated carbons in the same reaction [10]. Recent quasi in situ XPS data obtained from the same reaction using activated carbon felt as catalyst indicate that the presence of these species increases when the activity increases [19], supporting the proposed mechanism.

An oxidative pretreatment ( $2 \mathrm{vol} . \%$ of oxygen diluted in $\mathrm{He}$ for $3 \mathrm{~h}$ at $790 \mathrm{~K}$, i.e. the reaction temperature) of the OLC catalyst prior to the reaction did not modify the catalytic behaviour of the material and the catalyst showed a similar induction period with increasing ethylbenzene conversion at
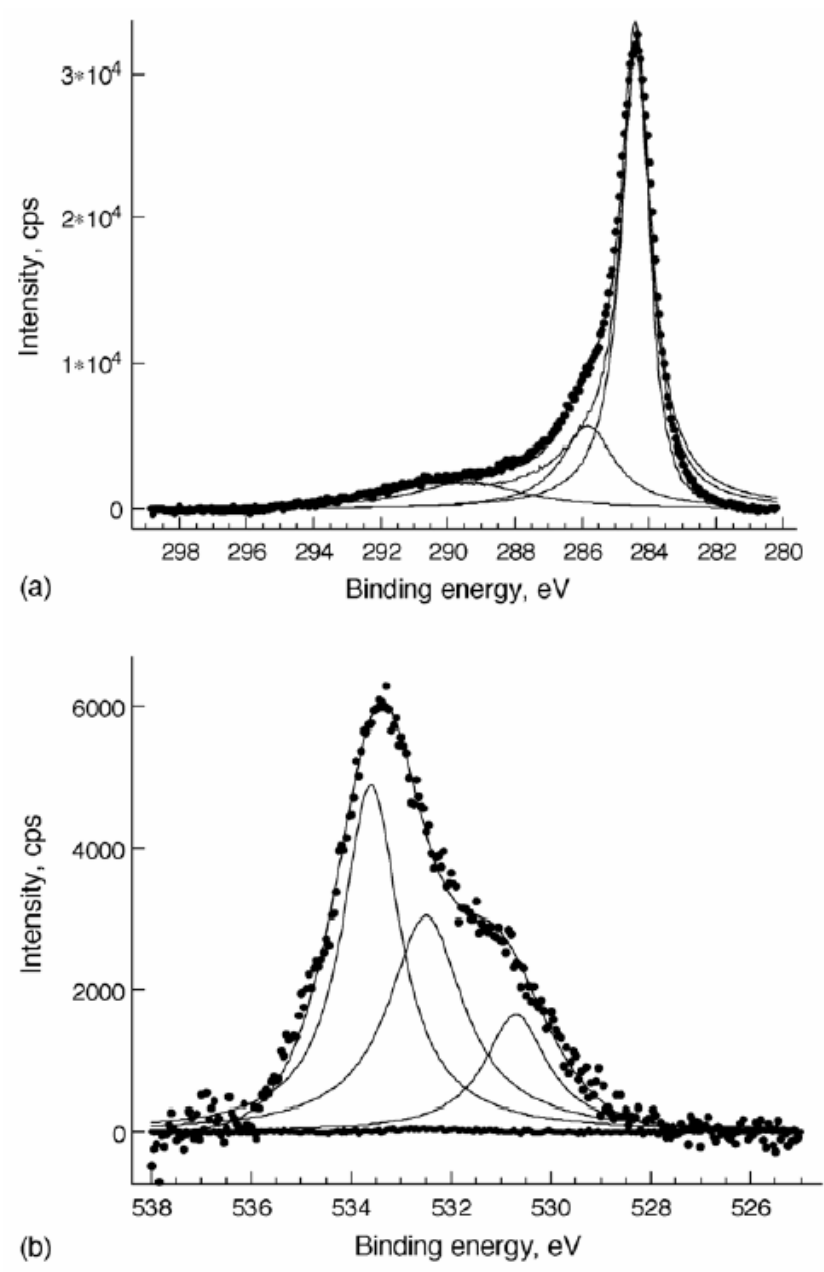

Fig. 4. (a) C $1 \mathrm{~s}$ XP spectra of OLC before (dashed line) and after (full line) and (b) O 1s XP spectra of OLC prior (solid line) and used (full circles).

the beginning of the reaction (not shown). However, the same oxygen treatment at $843 \mathrm{~K}$, i.e. $50 \mathrm{~K}$ higher than the reaction temperature, completely suppressed the induction period and the activated catalyst showed high and stable performances from the beginning of the reaction. This confirmed that the activation period could be related to the formation of oxygenated surface groups on stream. The higher temperature needed compared to the reaction temperature could be explained by the exothermicity of the ODH reaction. The observed differences in the initial catalytic behaviour of the different carbon materials have to be related to the formation of functional surface groups during the oxidative pretreatment or the induction period.

\subsection{Structure-activity relationship and reaction model}

The obtained results indicate that the performances of nanocarbons were directly and uniquely related to their microstructures. When compared to graphite and carbon nanotubes, the starting OLC material had a large surface abundance of graphitic $\left(\begin{array}{llll}0 & 0 & 0 & 1\end{array}\right)$ facets combined with a 
small abundance of edge/kink sites where the bending of the graphene layers occurs (blurred contrast in the TEM). These features are characteristic of a complete absence of surface oxygen functionalities. It was suggested that the strongly basic chinoidic surface functionalities, formed on the carbon catalyst during the induction period, were generated as resonance stabilized $\mathrm{C}=\mathrm{O}$ surface terminations of the edge/ kink regions of OLC. The superiority of OLC compared to other carbon forms derived from the higher number of active sites per unit weight at the steady-state and was related to the optimized distribution on this kind of nanocarbons, i.e. the sites required for oxygen activation (basal planes with metallic properties) and nucleophilic Brønsted basic $\mathrm{C}=\mathrm{O}$ groups (prismatic planes) with dehydrogenative properties $[16,13]$. Carbon materials can be considered as bi-functional catalysts with two different surface properties, metallic and oxidic, associated to two different surface phases.

A reaction model can be proposed for the ODH of ethylbenzene to styrene over $\mathrm{sp}^{2}$-hybridized carbon materials [16]: the reaction might occur via: (i) ethylbenzene adsorption at the graphite step edges; (ii) ethylbenzene reaction with the oxygenated species also located at the graphite step edges leading to the dehydrogenation of ethylbenzene to styrene by the scission of $\mathrm{C}-\mathrm{H}$ bond of its ethyl-group; (iii) the simultaneous transformation of the dehydrogenating oxygen species to hydroxyl groups which remains at the graphite edges; (iv) the styrene desorption; (v) gas-phase oxygen activation on the basal planes of the graphene layers; (vi) oxygen diffusion to the prismatic planes with the hydroxyl groups; (vii) reformation of the basic chinoidic oxygen functionalities from the activated oxygen and hydroxyl groups; and (viii) water desorption. In this reaction model, which follows a Langnuir-Hinshelwood formalism, both adsorbed ethylbenzene. In this model, adsorbed oxygen species (carbonyl/quinine) play a crucial role and it is in good agreement with the result reported by several authors using different activated carbons. Therefore, CNT and OLC show a similar mechanism and the activity of the samples can be indeed correlated to the presence of these oxygenated species.

\section{Conclusion}

Catalytic tests of different $\mathrm{sp}^{2}$-hybridized carbon structures evidenced the activity of $\mathrm{sp}^{2}$ carbon as catalyst for the selective ODH of ethylbenzene to styrene. It has been shown that the microstructure of the $\mathrm{sp}^{2}$-bound carbon material was of paramount importance in order to obtain high and stable efficiencies. The well-defined and oxygenfree surface of OLC rendered them a valuable model to derive criteria for designing efficient carbon catalysts and to put forward a reaction model. The OLC material showed the highest styrene yield $(62 \%)$ at the highest stable ethylben- zene conversion when compared to carbon nanotubes (54\%) and graphite $(44 \%)$, respectively, in correlation with a decreasing basal plane-edge/kink site ratio, whereas carbon black was completely combusted on stream. The perfectness of these nanocarbons provided enough stability towards oxidation and was essential for gas phase oxygen activation. We hope that tailoring other more available kinds of $\mathrm{sp}^{2}$-carbons into the desired target structure by synthetic and post-synthetic physicochemical procedures may allow to overcome the economic drawbacks of the OLC.

\section{Acknowledgements}

This work was performed in the frame of the European Laboratory for Catalysis and Surface Sciences (ELCASS). We thank the German Science Foundation (DFG) for financial support and Vladimir L. Kuznetsov from the Boreskov Institute of Catalysis, Novosibirsk for providing OLC samples.

\section{References}

[1] P.M. Ajayan, Chem. Mater. 11 (1999) 3862, and references therein.

[2] K.P. de Jong, J.W. Geus, Catal. Rev. Sci. Eng. 42 (2000) 481.

[3] P. Serp, M. Corrias, Ph. Kalck, Appl. Catal. A: Gen. 253 (2003) 337.

[4] NOVA Chemical Corporation Supplemental Financial and Product Information (2000) 10.

[5] D.H. James, W.M. Castor, in: H.-J. Arpe (Ed.), Ullmann's Encyclopedia of Industrial Chemistry, fifth ed., vol. A25, VCH Publishers, 1994 , p. 329.

[6] K.K. Kearby, in: O. Emmet (Ed.), Catalysis, vol. III, Reinhold, New York, 1955, p. 469.

[7] E.H. Lee, Catal. Rev. 8 (1973) 285

[8] F. Cavani, F. Trifiro, Appl. Catal. A: Gen. 133 (1995) 219.

[9] Guerrero-Ruiz, I. Rodriguez-Ramos, Carbon 32 (1994) 23.

[10] M.F.R. Pereira, J.J.M. Orfao, J.L. Figuereido, Appl. Catal. A: Gen. 184 (1-2) (1999) 153.

[11] M.F.R. Pereira, J.J.M. Orfao, J.L. Figuereido, Appl. Catal. A: Gen. 196 (1) (2000) 43.

[12] M.F.R. Pereira, J.J.M. Orfao, J.L. Figuereido, Appl. Catal. A: Gen. 218 (1-2) (2001) 307.

[13] G. Mestl, N.I. Maksimova, N. Keller, V.V. Roddatis, R. Schlögl, Ang. Chem. Int. Ed. 40 (11) (2001) 2066.

[14] N. Keller, N.I. Maksimova, V.V. Roddatis, M. Schur, G. Mestl, Y.V. Butenko, V.L. Kuznetsov, R. Schlögl, Angew. Chem. Int. Ed. 41 (11) (2002) 1885

[15] V.L. Kuznetsov, A.L. Chuvilin, Y.V. Butenko, I.Y. Malkov, V.M. Titov, Chem. Phys. Lett. 222 (1994) 343.

[16] N.I. Maksimova, Ph.D. thesis, Technical University Berlin, Germany, 2003.

[17] H. Ago, T. Kugler, F. Cacialli, W.R. Salaneck, M.S.P. Shaffer, A.H. Windle, R.H. Friend, J. Phys. Chem. B 103 (1999) 8116.

[18] R.P. Vidano, D.B. Fishbach, L.J. Willis, T.M. Loehr, Solid State Commun. 39 (1981) 423

[19] J.A. Maciá-Agulló, D. Cazorla-Amorós, A. Linares-Solano, U. Wild, D.S. Su, R. Schlögl, Catal. Today. 102-103 (2005) 248-253. 